\title{
Non-small cell lung cancer-small cell lung cancer transformation as mechanism of resistance to tyrosine kinase inhibitors in lung cancer
}

\author{
Barbara Rath, Adelina Plangger, Gerhard Hamilton \\ Department of Vascular Surgery, Medical University of Vienna, Vienna A-1090, Austria.
}

Correspondence to: Dr. Gerhard Hamilton, Medical University of Vienna, Department of Vascular Surgery, AKH 8H, Spitalgasse 23, Vienna A-1090, Austria. E-mail: gerhard.hamilton@meduniwien.ac.at

\begin{abstract}
How to cite this article: Rath B, Plangger A, Hamilton G. Non-small cell lung cancer-small cell lung cancer transformation as mechanism of resistance to tyrosine kinase inhibitors in lung cancer. Cancer Drug Resist 2020;3:171-8.

http://dx.doi.org/10.20517/cdr.2019.85
\end{abstract}

Received: 29 Sep 2019 First Decision: 30 Dec 2019 Revised: 13 Jan 2020 Accepted: 18 Feb 2020 Available online: 28 Feb 2020

Science Editor: Lee M. Graves Copy Editor: Jing-Wen Zhang Production Editor: Tian Zhang

\begin{abstract}
Mutated or rearranged driver kinases in non-small cell lung cancer (NSCLC) cells are clinically amenable to treatment with tyrosine kinase inhibitors (TKIs) resulting in prolonged survival and significant benefit compared to cytotoxic chemotherapy. The most frequent genomic alterations are observed for epidermal growth factor receptor and anaplastic lymphoma kinase, which can be blocked by a range of specific TKIs in sequence. In clinics, resistance to TKIs emerges after approximately one year and comprises secondary mutations of the kinases (on-target) or alternative pathways circumventing the original kinase (off-target) alterations. A special feature of NSCLC is the occurrence of histological transformation to small cell lung cancer (SCLC) in up to $14 \%$ of cases, which, in general, is accompanied by resistance to the original TKIs. SCLC transformed tumors may be treated with the classical platinum/etoposide regimen but thus far there are no definitive guidelines. Four transformed pleural SCLC lines in our lab indicate the presence of a gradual NSCLC-SCLC shift with overlapping drug sensitivities. In conclusion, the treatment of NSCLC-SCLC transformed cancer cells would need a better chemosensitivity assessment using functional genomics to guide further therapy.
\end{abstract}

Keywords: Lung cancer, non-small cell lung cancer, small cell lung cancer, transformation, epidermal growth factor receptor, tyrosine kinase inhibitor, chemotherapy, drug resistance

\section{INTRODUCTION}

The tyrosine kinase inhibitors (TKIs) have revolutionized the treatment of lung cancer patients with respective mutations of so-called driver kinases and have demonstrated superior clinical activity compared 
to cytotoxic chemotherapy ${ }^{[1]}$. Furthermore, the inhibition of kinase signaling pathways are less toxic to normal tissues, resulting in considerably lower side effects ${ }^{[2]}$. Previously, chemotherapy using cisplatin in combination with etoposide, pemetrexed, or docetaxel was the standard of care for metastatic nonsmall cell lung cancer (NSCLC) and revealed five-year survival rates below $10 \%{ }^{[3]}$. In the last decade, the proliferation of a part of the NSCLC cells was found to depend on a range of mutated or rearranged tyrosine kinases that could be targeted by specific inhibitors, which are preferentially directed to the ATP binding pockets of these enzymes. The most important altered kinases comprise epidermal growth factor receptor (EGFR) with a frequency of $15 \%-20 \%$, HER 2 with $2 \%$, rearranged anaplastic lymphoma kinase (ALK) with $2 \%-7 \%$, and rearranged ROS 1 with a frequency of $1.7 \%^{[1]}$. Ligand binding to EGFR induces dimerization with other ERBB family members and the resulting receptor phosphorylation activates downstream RAS-RAF-MEK-ERK and RAS-PI3K-PTEN-AKT-mTOR pathways, leading to increased proliferation $^{[4]}$. Although these mutations and rearrangements of EGFR, ALK, and ROS1 occur in a higher number of lung cancer cases, several other kinases show rare mutations and a wide range of different fusion genes and associated partners. Mutations result in constitutive activation of the kinases and rearrangements may activate kinases through the promoter function of the fusion partners. Historically, the EGFR TKIs erlotinib and gefitinib showed clinical activity against the tumors exhibiting corresponding mutations and a similar activity was proved for the TKI crizotinib and ALK-rearranged cancers ${ }^{[1]}$. NSCLCs become resistant to first-line EGFR-TKIs, within a median progression-free survival (PFS) of 9-13 month ${ }^{[5]}$. Recently, the use of frontline osimertinib, a third-generation TKI, has resulted in a PFS of 19 months and a response rate of approximately $80 \%{ }^{[6]}$. In detail, pretreated T790M-mutant patients were reported to yield a median overall survival (OS) of 26.8 months and 12-, 24-, and 36-month survival rates of $80 \%, 55 \%$, and $37 \%$ in response to osimertinib, respectively ${ }^{[7]}$. However, $20 \%-30 \%$ of EGFR mutated patients do not respond at all or only for a short time because of intrinsic resistance existing before treatment ${ }^{[6]}$. Furthermore, coalterations in MET or other genes of the MAPK, PI3K, and Wnt/ $\beta$-catenin signaling pathways and in cell cyle genes are linked to a poor response to EGFR-TKIs ${ }^{[8]}$.

Kinase inhibition exerts a strong pressure for the tumor cells to acquire resistance to TKIs through further kinase mutations or alternative pathways [Figure 1]. Therefore, despite the prominent anticancer activities of the TKIs, resistance develops invariably within approximately 12-18 months and the tumors relapse ${ }^{[6]}$. Further preclinical drug development provided access to second- and third-line TKIs that could be administered in sequence to inhibit the serially mutated kinases in order to prolong survival [Figure 1]. For example, erlotinib and gefinitib may be followed by afatinib or osimertinib for mutated EGFR and alectinib or brigatinib by lorlatinib or other ALK-directed agents. Crizotinib has been largely abandoned as a firstline agent due to its low intracranial activity. These agents are active against resistance caused by ontarget mutations, i.e., further genetic modifications that alter the target structures of the respective kinases themselves. In contrast, non-target mutations result in activation or upregulation of other proliferationstimulating pathways which bypass the dependence on the original mutated target and lead to tumor progress. Thus, the series of second- and third-line EGFR or ALK TKIs is eventually rendered inactive by activation of alternative kinases and, in the case of NSCLC, by a histological switch to a small cell lung cancer (SCLC) phenotype, which is not susceptible to the original TKIs ${ }^{[9]}$. Although formerly regarded to be rare with $1 \%-3 \%$ of cases, it became clear that NSCLC-SCLC transformation is more frequent with an incidence of up to $14 \%$ of cases and the resulting SCLC tumors exhibit varying characteristics and chemosensitivities $^{[9]}$.

\section{RESISTANCE TO MUTATED EGFR-DIRECTED TKIS}

EGFR-activating mutations are detected in approximately $15 \%-20 \%$ of NSCLC patients ${ }^{[10]}$. Accordingly, the available EGFR-directed TKIs are administered either in sequence or with the most active inhibitor, osimertinib, as first-line agent. However, tumor progress after the application of a sequence of TKIs is complex and not fully characterized. Repeat tissue or liquid biopsies during therapy have demonstrated 


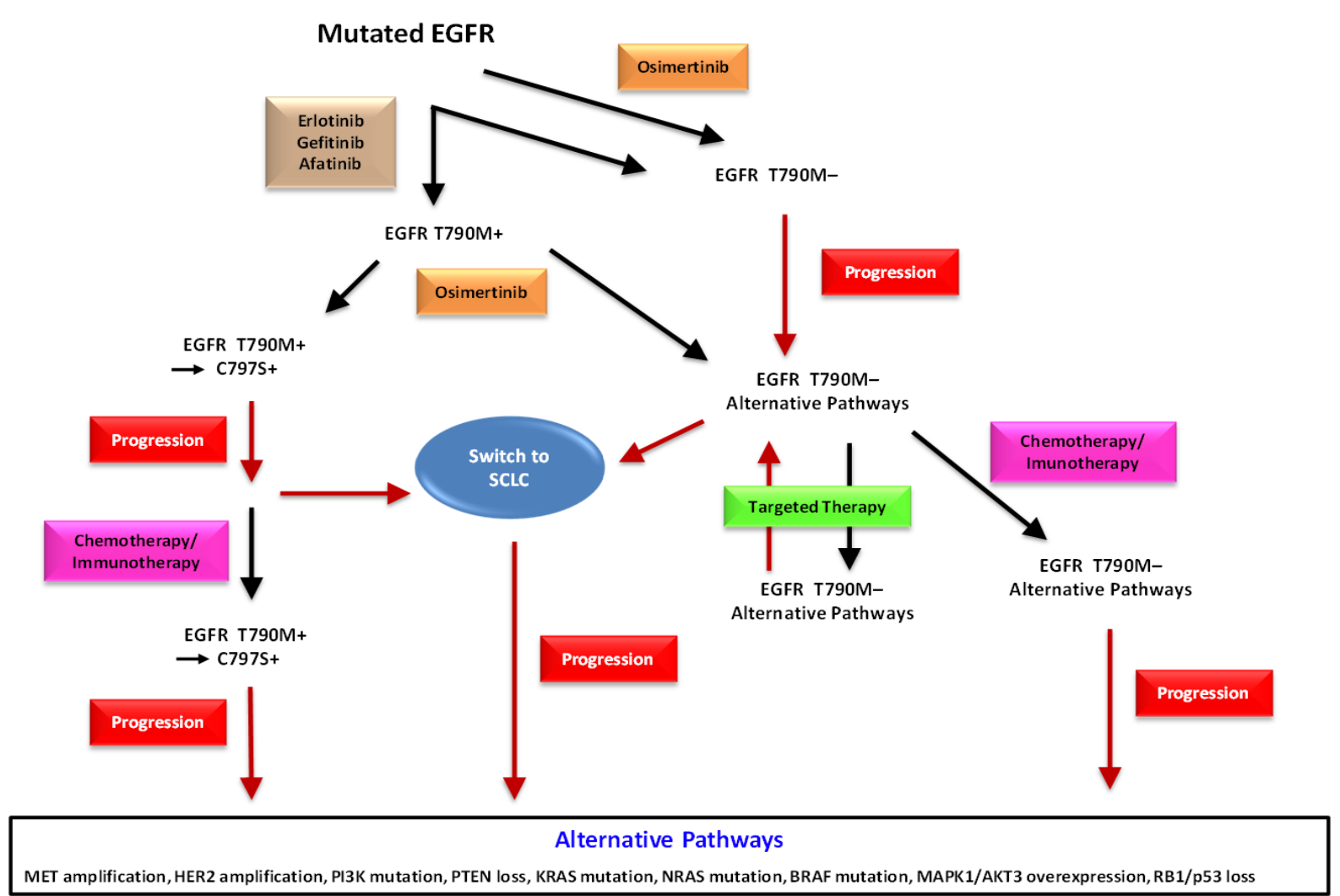

Figure 1. This scheme represents the sequence of therapeutic regimens in EGFR-mutated NSCLC. Tumors are treated with first-line agents erlotinib, gefitinib, or afatinib. Alternatively, the second-line osimertinib is applied directly or as actual second-line therapy. C797S may appear as further mutation of EGFR followed by progress and eventually by rescue chemotherapy. First-line osimertinib is rendered inactive by loss of T790M and the patients may be further treated by targeted agents against alternative pathway modulators and, upon progress, by rescue chemotherapy. Switch to a SCLC histotype may occur from both branches of therapy and result in cancer resistance and progress. SCLC: small cell lung cancer; EGFR: epidermal growth factor receptor; NSCLC: non-small cell lung cancer

multiple mechanisms of resistance, including secondary and tertiary EGFR mutations; bypass pathways such as c-MET or HER2 amplification; mutations of RAS, BRAF, PIK3CA, and other growth regulators; and novel fusion events and histological transformation ${ }^{[8]}$. The most common EGFR mutations are deletions in exon 19 (54\%) and substitution of leucine with arginine at codon 858 (L858R, 41\%), aside from a range of minor mutations ${ }^{[11]}$. Twenty percent of NSCLC patients with TKI resistance are related to $c-M E T$ gene amplification, which is not correlated with the presence of T790M mutation ${ }^{[12]}$. The $c-M E T$ gene amplification activates the ERBB3-PI3K signaling pathway directly. Patients sensitive to EGFR TKIs eventually progress after approximately 9-11 months compared to five months under chemotherapy. More than $60 \%$ of patients reveal resistance to first- or second-line inhibitors through a threonine-tomethionine substitution at the EGFR position $790(\mathrm{~T} 790 \mathrm{M})$ in the ATP binding pocket of EGFR exon $20^{[11,13]}$. Metastatic EGFR T790M-positive NSCLC is treated with osimertinib, which is now approved for first-line treatment of EGFR-mutant lung cancer. Osimertinib binds irreversibly to the cysteine-797 residue at the ATP binding site of the EGFR but is subsequently rendered inactive by a further C797S mutation, against which several drugs are under development ${ }^{[14]}$. First-line application of osimertinib seems to result in different mechanisms of resistance, including a higher frequency of NSCLC-SCLC transformations. Resistance mechanisms involving $c-M E T$ and HER2 amplification can be treated with crizotinib, which in addition to ALK also inhibits c-MET, and the HER2-targeting TKI afatinib, which blocks all types of ERBBs, respectively ${ }^{[1,5,8]}$. Signal transduction inhibitors and a host of novel agents interfering with alternative pathways are under preclinical and clinical development. 


\section{NSCLC TRANSFORMATION TO SCLC}

When TKI resistance develops, patients generally undergo either a second tissue or a first liquid biopsy to determine the molecular mechanisms of refractoriness in order to guide the next treatment step and to predict the respons $\mathrm{e}^{[13,15]}$. Resistance to EGFR inhibitors through histological transformation of lung EGFRmutant adenocarcinoma to SCLC has been reported in $3 \%-14 \%$ of cases in repeated biopsies series ${ }^{[1,15-17]}$. Typically, tumor tissues of lung adenocarcinoma patients with SCLC histology show expression of the typical markers synaptophysin, chromogranin, and CD56. In a recent study, 206 biopsies of EGFR TKIresistant adenocarcinoma patients showed 21 SCLC transformed cases ${ }^{[17]}$. Patients experiencing SCLC transformation after third-generation EGFR TKI treatment (e.g., osimertinib) were included in the analysis. Many other isolated cases of histological transformation to SCLC under EGFR TKI treatment have been reported since $2006^{[15,18,19]}$. Biopsies from 71 patients with acquired resistance to osimertinib showed histological transformation in $14 \%$ overall and in $19 \%$ of samples after first-line osimertinib, in contrast to liquid biopsy-based results, which reported lower percentages of transformation ${ }^{[20,21]}$. NSCLCSCLC transformation can be monitored in plasma samples by detection of increases of TP53 mutations and, more specifically, of copy number changes of SCLC-associated markers MYCL1, SOX2, and SOX $4^{[22]}$. Accordingly, with $18 \%$ of cases, tertiary EGFR mutations were more common in patients treated with laterline osimertinib than first-line osimertinib with $6 \%{ }^{[22]}$.

Transformation of NSCLC into SCLC may be due to a combined histology or a true switch of the histotype ${ }^{[9]}$. However, almost every transformed SCLC retained the original EGFR activating mutation of the parent adenocarcinoma and, correspondingly, strong and durable response to EGFR TKIs were found initially. These data are in favor of a true transformation and exclude a possible preexistent mixed histology ${ }^{[19,23,24]}$. The underlying mechanism of this histological NSCLC-SCLC transition is not clear. However, loss of RB1 was proven in a series of 11 cases of transformed EGFR mutant NSCLC specimens ${ }^{[9,24,25]}$. Upon complete inactivation of the tumor suppressor genes RB1 and TP53 at baseline, the clonal branching of SCLC cells from adenocarcinoma became visible before the TKI-start and the risk of SCLC transformation was increased $>40$ times $^{[17]}$. Time to transformation from the initial advanced NSCLC ranged from 2 to 60 months (median $=17.8$ months) and from TKI-start between 1.3 and 53.4 months (median $=15.8$ months) ${ }^{[26]}$. The frequent mutations in TP53, RB1, and PIK3CA are also typical of classic SCLC ${ }^{[27]}$. However, RB1 and TP53 loss seems to be necessary, but not sufficient ${ }^{[16,17,28]}$. This tumor suppressor inactivation is accompanied by decreased EGFR expression, despite the persistence of the EGFR activating mutation ${ }^{[9,24]}$. SCLC is thought to originate from neuroendocrine cells localized within the airways wall, while adenocarcinoma seems to be more related to alveolar type II cells ${ }^{[9,29]}$. However, the development of SCLC from alveolar type II cells was detected in mouse models ${ }^{[30]}$. Cellular plasticity between NSCLC and SCLC support the concept of a mutual origin from pluripotent alveolar cells ${ }^{[9,19]}$. The combination of EGFR TKI treatment and genetic and epigenetic modifications, such as RB1 loss and EGFR down-regulation, could switch NSCLCs towards a SCLC histotype ${ }^{[9]}$. SCLC transformation from NSCLC has rarely been observed in EGFR-wild type lung cancers or during ALK-targeted therapy and programed cell death-1 (PD-1/L)-directed immunotherapy ${ }^{[31]}$. In addition to transformation to SCLC, histological switch to other NSCLC subtypes such as squamous cell carcinoma, large-cell neuroendocrine carcinoma, or sarcoma after TKI resistance have all been published ${ }^{[32]}$.

\section{TREATMENT OF NSCLC-SCLC TRANSFORMED TUMORS}

NSCLC-SCLC transformation complicates the choice of an efficient therapeutic strategy. Initial reports indicated that the prognosis of transformed SCLC is poor and standard chemotherapy for primary SCLC appeared to be ineffective ${ }^{[26,33]}$. However, recent studies have reported favorable outcome after conventional systemic chemotherapy as applied for SCLC ${ }^{[25,34,35]}$. Platinum-etoposide was associated with an overall response rate (ORR) of $54 \%$ and a median PFS of 3.4 months $^{[26]}$. Thus, patients with SCLC combined with 

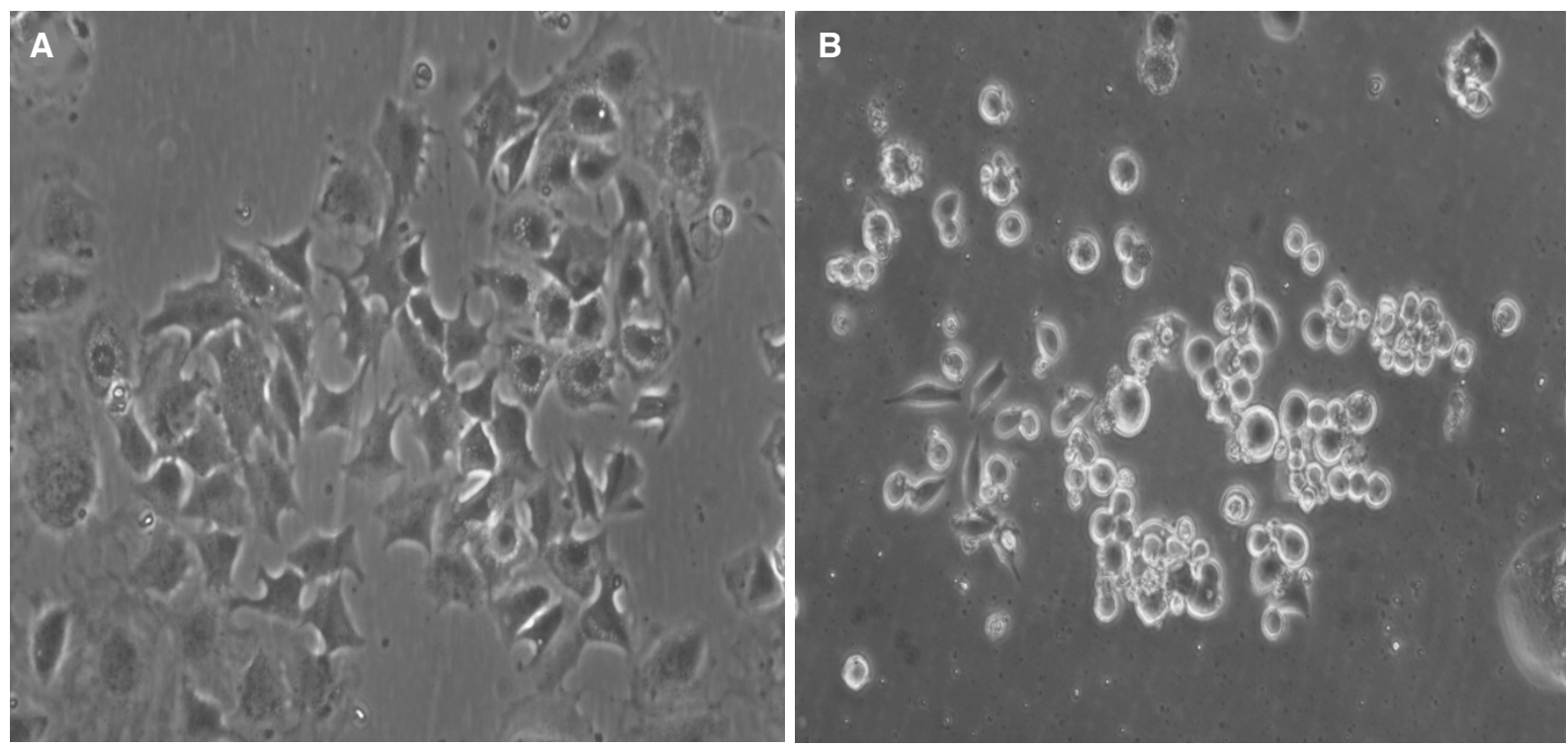

Figure 2. NSCLC-SCLC transformed pleural cell lines display either a phenotype still similar to a typical adenocarcinoma as in the case of BH15 (A) or a classical SCLC phenotype as in the case of BH2O (B). Both cell lines express SCLC markers. NSCLC: non-small cell lung cancer; SCLC: small cell lung cancer

NSCLC and SCLC transformed from adenocarcinoma should be offered standard therapies for SCLC ${ }^{[26]}$. Administration of immunotherapy with PD-1 or PD-L1 inhibitors for 17 patients with NSCLC-SCLC transformation showed no responders. Patients progressing on platinum-etoposide chemotherapy were treated with taxanes, yielding an ORR of $50 \%$ and a median PFS of 2.7 months. In vitro, osimertinibresistant cells with SCLC transformation were more sensitive to paclitaxel compared with osimertinibsensitive cells ${ }^{[23,24]}$. The median OS from diagnosis was 31.5 months, and median OS from transformation was 10.9 months. Following transformation to SCLC, central nervous system metastases were observed in $64 \%$ patients. In most cases (84\%), the EGFR-mutant tumors maintain the same mutation after SCLC transformation but show additional loss or mutation in TP53 and $R B 1$ genes, which are frequently seen in SCLC ${ }^{[17]}$. Several clinical cases of SCLC transformation after osimertinib treatment were reported to respond well to platinum-based doublet chemotherapy ${ }^{[36]}$. However, the short median PFS and OS after histological transformation indicate that the currently used therapeutic protocols are inefficient. The respective study by Marcoux et al ${ }^{[26]}$ is corroborated by a series of 39 TKI-treated SCLC-transformed NSCLCs and a retrospective European cohort of 48 such patients in respect to response rate and OS $^{[32]}$. Further research efforts are focused on trying to assess SCLC transformation by the use of non-invasive biomarkers such as serum pro-gastrin-releasing peptide, neuron-specific enolase, and liquid biopsy ${ }^{[22,37]}$.

\section{PHENOTYPE OF NSCLC-SCLC TRANSFORMED TUMOR CELLS}

Increased expression of neuroendocrine markers and decreased EGFR expression were detected in TKI resistant SCLC transformed cancers compared with resistant NSCLCs ${ }^{[24]}$. Usually, the cells change from an adherent widespread growth pattern to typical rounded suspension cultures under tissue conditions upon SCLC transformation [Figure 2]. Thus, following SCLC transformation, cancer cells became insensitive to EGFR-TKIs partly by downregulating the expression of EGFR protein and not by acquiring a secondary EGFR-mutation such as $T 790 M^{[9,19,38]}$. However, 4 out of 19 cases retained the T790M mutation after transformation to SCLC ${ }^{[17,25]}$. Additionally, the RNA profile of SCLC-transformed NSCLC show a mixed transcriptome composed of classical SCLC features and a subset of mRNA more typically found in adenocarcinoma ${ }^{[24]}$. Tumor heterogeneity with concurrent patchy SCLC transformation and T790M EGFR resistance mutation in the same patients have been described ${ }^{[39]}$. For example, a patient who experienced 
resistance to therapy with afatinib through a histotype transformation to SCLC exhibited a locally confined chemosensitive switch of a part of the tumor ${ }^{[9,40]}$. Although EGFR mutations were identified in SCLC, these patients have had variable responses to EGFR inhibitors most likely related to the loss of EGFR expression at the protein level ${ }^{[2,41,42]}$. The transformed tumors are not always completely insensitive to EGFR-TKIs, as $52 \%$ of patients in the North American cohort received TKI therapy after transformation and a few cases showed clinical benefit from this treatment ${ }^{[26]}$. Subsequent re-challenge with the first-line TKIs has been tried as salvage treatment after progression of chemotherapy, yielding modest activity with a median PFS of 2.8 or 6.5 months following gefitinib or erlotinib treatment, respectively ${ }^{[43]}$.

\section{CONCLUSION}

The most efficient therapy for SCLC-transformed NSCLC cells is not clear. The analysis of RNA expression of two NSCLC-SCLC transformed cell lines revealed a unique gene expression pattern in-between those of NSCLC and classical SCLC cells ${ }^{[29]}$. The morphologies of EGFR-mutant SCLCs are similar to those of typical SCLCs, but gene expression is different. These results may imply that EGFR-mutant SCLC has more phenotypic and functional plasticity than typical SCLC. The foundation treatment for SCLC is still chemotherapy but the response is far from satisfactory. In our lab, we have obtained four SCLC transformed cell lines from pleural effusions of NSCLC patients progressing after several TKI treatment periods. These cell lines exhibit a range of phenotypes resembling from adherent NSCLC with SCLC marker expression (BH15) to classical appearance of SCLC lines with small size cells in suspension culture (BH20). Whereas $\mathrm{BH} 15$ exhibits high resistance to cisplatin, $\mathrm{BH} 20$ is fully chemosensitive to the chemotherapeutics employed in therapy of SCLC, such as cisplatin, topotecan, and epirubicin (data not shown). However, for the BH20 SCLC line, tumorospheres were observed in tissue culture, which has been found to exhibit broad chemoresistance ${ }^{[44]}$. Surprisingly, the single approved drug for relapsed SCLC, topotecan, was not used in second-line treatment of advanced transformed SCLC, as well as the alternative epirubicin-based regimen but, instead, taxanes were administered. The example of $\mathrm{BH} 15$ demonstrates that tumors more similar to NSCLC after a partial change of the histotype may exhibit high resistance to cisplatin and are expected to respond poorly to the cisplatin/etoposide SCLC standard therapy. In such cases, second-line therapeutics such as topotecan or cyclosphosphamide/epirubicin/vincristine should be considered. Therapy of SCLC urgently needs new modalities of treatment such as inhibition of prostaglandin E synthase-1, which increases sensitivity to cisplatin and EGFR TKIs simultaneously ${ }^{[45]}$. However, functional testing of tumor cells may be essential to provide the best available chemotherapy for the NSCLC-SCLC transformed TKI-resistant tumors ${ }^{[46]}$.

\section{DECLARATIONS}

\section{Acknowledgments}

We wish to thank Dr. T. Hohenheim for continuous endorsement.

\section{Authors' contributions}

Participated in study design and figures and helped to draft the manuscript: Rath B, Plangger A

Read and approved the final manuscript: Rath B, Plangger A, Hamilton G

\section{Availability of data and materials}

Not applicable.

\section{Financial support and sponsorship}

None.

\section{Conflicts of interest}

All authors declared that there are no conflicts of interest. 


\section{Ethical approval and consent to participate}

Not applicable.

\section{Consent for publication}

Not applicable.

\section{Copyright}

(c) The Author(s) 2020.

\section{REFERENCES}

1. Recondo G, Facchinetti F, Olaussen KA, Besse B, Friboulet L. Making the first move in EGFR-driven or ALK-driven NSCLC: firstgeneration or next-generation TKI? Nat Rev Clin Oncol 2018;15:694-708.

2. Bhullar KS, Lagarón NO, McGowan EM, Parmar I, Jha A, et al. Kinase-targeted cancer therapies: progress, challenges and future directions. Mol Cancer 2018;17:48.

3. Zappa C, Mousa SA. Non-small cell lung cancer: current treatment and future advances. Transl Lung Cancer Res 2016;5:288-300.

4. Sukrithan V, Deng L, Barbaro A, Cheng H. Emerging drugs for EGFR-mutated non-small cell lung cancer. Expert Opin Emerg Drugs 2019;24:5-16.

5. Camidge DR, Pao W, Sequist LV. Acquired resistance to TKIs in solid tumours: learning from lung cancer. Nat Rev Clin Oncol 2014;11:473-81.

6. Santoni-Rugiu E, Melchior LC, Urbanska EM, Jakobsen JN, Stricker K, et al. Intrinsic resistance to EGFR-tyrosine kinase inhibitors in EGFR-mutant non-small cell lung cancer: differences and similarities with acquired resistance. Cancers (Basel) 2019;11.

7, Ahn MJ, Tsai CM, Shepherd FA, Bazhenova L, Sequist LV, et al. Osimertinib in patients with T790M mutation-positive, advanced non-small cell lung cancer: Long-term follow-up from a pooled analysis of 2 phase 2 studies. Cancer 2019;125:892-901.

8. Blakely CM, Watkins TBK, Wu W, Gini B, Chabon JJ, et al. Evolution and clinical impact of co-occurring genetic alterations in advanced-stage EGFR-mutant lung cancers. Nat Genet 2017;49:1693-704.

9. Oser MG, Niederst MJ, Sequist LV, Engelman JA. Transformation from non-small-cell lung cancer to small-cell lung cancer: molecular drivers and cells of origin. Lancet Oncol 2015;16:e165-72.

10. Murtuza A, Bulbul A, Shen JP, Keshavarzian P, Woodward BD, et al. Novel third-generation EGFR tyrosine kinase inhibitors and strategies to overcome therapeutic resistance in lung cancer. Cancer Res 2019;79:689-98.

11. Sequist LV, Waltman BA, Dias-Santagata D, Digumarthy S, Turke AB, et al. Genotypic and histological evolution of lung cancers acquiring resistance to EGFR Inhibitors. Sci Transl Med 2011;3:75ra26.

12. Jiao Q, Bi L, Ren Y, Song S, Wang Q, et al. Advances in studies of tyrosine kinase inhibitors and their acquired resistance. Mol Cancer 2018;17:36.

13. Hochmair MJ, Buder A, Schwab S, Burghuber OC, Prosch H. Liquid-biopsy-based identification of EGFR T790M mutation-mediated resistance to afatinib treatment in patients with advanced EGFR mutation-positive NSCLC, and subsequent response to osimertinib. Target Oncol 2019;14:75-83.

14. Thress KS, Paweletz CP, Felip E, Cho BC, Stetson D, et al. Acquired EGFR C797S mutation mediates resistance to AZD9291 in nonsmall cell lung cancer harboring EGFR T790M. Nat Med 2015;21:560-2.

15. Tomasello C, Baldessari C, Napolitano M, Orsi G, Grizzi G, et al. Resistance to EGFR inhibitors in non-small cell lung cancer: clinical management and future perspectives. Crit Rev Oncol Hematol 2018;123:149-61.

16. Yu HA, Suzawa K, Jordan E, Zehir A, Ni A, et al. Concurrent alterations in EGFR-mutant lung cancers associated with resistance to EGFR kinase inhibitors and characterization of MTOR as a mediator of resistance. Clin Cancer Res 2018;24:3108-18.

17. Lee JK, Lee J, Kim S, Kim S, Youk J, et al. Clonal history and genetic predictors of transformation into small-cell carcinomas from lung adenocarcinomas. J Clin Oncol 2017;35:3065-74.

18. Ahn S, Hwang SH, Han J, Choi YL, Lee SH, et al. Transformation to small cell lung cancer of pulmonary adenocarcinoma: clinicopathologic analysis of six cases. J Pathol Transl Med 2016;50:258-63.

19. Dorantes-Heredia R, Ruiz-Morales JM, Cano-García F. Histopathological transformation to small-cell lung carcinoma in non-small cell lung carcinoma tumors. Transl Lung Cancer Res 2016;5:401-12.

20. Schoenfeld AJ, Chan JM, Rizvi H, Rekhtman N, Daneshbod Y, et al. Tissue-based molecular and histological landscape of acquired resistance to osimertinib given initially or at relapse in patients with EGFR-mutant lung cancers. J Clin Oncol 2019;37:9028.

21. Girard N. Optimizing outcomes and treatment sequences in EGFR mutation-positive non-small-cell lung cancer: recent updates. Future Oncol 2019;15:2983-97.

22. Tsui DWY, Murtaza M, Wong ASC, Rueda OM, Smith CG, et al. Dynamics of multiple resistance mechanisms in plasma DNA during EGFR-targeted therapies in non-small cell lung cancer. EMBO Mol Med 2018;10.

23. Ferrer L, Giaj Levra M, Brevet M, Antoine M, Mazieres J, et al. A brief report of transformation from NSCLC to SCLC: molecular and therapeutic characteristics. J Thorac Oncol 2019;14:130-4.

24. Niederst MJ, Sequist LV, Poirier JT, Mermel CH, Lockerman EL, et al. RB loss in resistant EGFR mutant lung adenocarcinomas that transform to small-cell lung cancer. Nat Comm 2015;6:6377.

25. van Meerbeeck JP, Fennell DA, De Ruysscher DK. Small-cell lung cancer. Lancet 2011;378:1741-55.

26. Marcoux N, Gettinger SN, O’Kane G, Arbour KC, Neal JW, et al. EGFR-mutant adenocarcinomas that transform to small-cell lung 
cancer and other neuroendocrine carcinomas: clinical outcomes. J Clin Oncol 2019;37:278-85.

27. George J, Lim JS, Jang SJ, Cun Y, Ozretić L, et al. Comprehensive genomic profiles of small cell lung cancer. Nature 2015;524:47-53.

28. Kim Y, Lee B, Shim JH, Lee SH, Park WY, et al. Concurrent genetic alterations predict the progression to target therapy in EGFRmutated advanced NSCLC. J Thorac Oncol 2019;14:193-202.

29. Lin CA, Yu SL, Chen HY, Chen HW, Lin SU, et al. EGFR-mutant SCLC exhibits heterogeneous phenotypes and resistance to common antineoplastic drugs. J Thorac Oncol 2019;14:513-26.

30. Sutherland KD, Proost N, Brouns I, Adriaensen D, Song JY, et al. Cell of origin of small cell lung cancer: inactivation of Trp53 and $\mathrm{Rb} 1$ in distinct cell types of adult mouse lung. Cancer Cell 2011;19:754-64.

31. Park CK, Oh IJ, Kim YC. Is transformed small cell lung cancer (SCLC) different from de novo SCLC? Transl Cancer Res 2019;8:346-9.

32. Shao Y, Zhong DS. Histological transformation after acquired resistance to epidermal growth factor tyrosine kinase inhibitors. Int $\mathrm{J}$ Clin Oncol 2018;23:235-42.

33. Roca E, Gurizzan C, Amoroso V, Vermi W, Ferrari V, et al. Outcome of patients with lung adenocarcinoma with transformation to small-cell lung cancer following tyrosine kinase inhibitors treatment: a systematic review and pooled analysis. Cancer Treat Rev 2017;59:117-22.

34. Sharma S, Kwiat C, Das K, Esposito MJ, Barnaby K, et al. Small cell transformation: an increasingly common mechanism of resistance in EGFR-mutated lung cancer. J Target Ther Cancer 2018.

35. Suda K, Murakami I, Sakai K, Mizuuchi H, Shimizu S, et al. Small cell lung cancer transformation and T790M mutation: complementary roles in acquired resistance to kinase inhibitors in lung cancer. Sci Rep 2015;5:14447.

36. Ham JS, Kim S, Kim HK, Byeon S, Sun JM, et al. Two cases of small cell lung cancer transformation from EGFR mutant adenocarcinoma during AZD9291 treatment. J Thorac Oncol 2016;11:e1-4.

37. Oh HJ, Park HY, Kim KH, Park CK, Shin HJ, et al. Progastrin-releasing peptide as a diagnostic and therapeutic biomarker of small cell lung cancer. J Thorac Dis 2016;8:2530-7.

38. Santoni-Rugiu E. Clinical outcomes provide new insights into transformation to small-cell lung cancer of pulmonary EGFR-mutant adenocarcinoma. Precis Cancer Med 2019;2:5.

39. Farago AF, Piotrowska Z, Sequist LV. Unlocking the mystery of small-cell lung transformations in EGFR mutant adenocarcinoma. J Clin Oncol 2017;35:2987-8.

40. Manca P, Russano M, Pantano F, Tonini G, Santini D. Change from lung adenocarcinoma to small cell lung cancer as a mechanism of resistance to afatinib. Oncotarget 2017;8:59986-90.

41. Araki J, Okamoto I, Suto R, Ichikawa Y, Sasaki J. Efficacy of the tyrosine kinase inhibitor gefitinib in a patient with metastatic small cell lung cancer. Lung Cancer 2005;48:141-4.

42. Shiao TH, Chang YL, Yu CJ, Chang YC, Hsu YC, et al. Epidermal growth factor receptor mutations in small cell lung cancer: a brief report. J Thorac Oncol 2011;6:195-8.

43. Lee S, Joo J, Kwak M, Sohn K, Chon S. Role of chemotherapy with epidermal growth factor receptor-tyrosine kinase inhibitor (EGFR-TKI) rechallenge in small cell transformation after EGFR-TKI failure: a case report. Onco Targets Ther 2018;11:3943-7.

44. Hamilton G, Rath B. Role of circulating tumor cell spheroids in drug resistance. Cancer Drug Resist 2019;2:762-72.

45. Terzuoli E, Costanza F, Ciccone V, Ziche M, Morbidelli L, et al. mPGES-1 as a new target to overcome acquired resistance to gefitinib in non-small cell lung cancer cell lines. Prostaglandins Other Lipid Mediat 2019;143:106344.

46. Hamilton G, Rath B, Plangger A, Hochmair M. Implementation of functional precision medicine for anaplastic lymphoma kinaserearranged non-small lung cancer. Precis Cancer Med 2019;2:19. 